\title{
A BIOGRAPHY OF THE POINT CONTACT FIXATOR (PC-FIX)
}

\author{
S. Tepic ${ }^{1,2}$ and S. Bresina ${ }^{1,2, *}$ \\ ${ }^{1}$ Scyon Orthopaedics AG, Zurich, Switzerland \\ ${ }^{2}$ Kyon AG, Zurich, Switzerland
}

\begin{abstract}
Starting in the mid-eighties, the AO (from the German "Arbeitsgemeinschaft für Osteosynthesefragen") Research Institute (ARI), Davos, Switzerland together with the commercial partners of the AO Foundation embarked on a decade-long project to design, develop, test in experimental animals and human clinical trials as well as bring to clinical use a new system for surgical osteosynthesis. The new plating system, what became known as the Point Contact Fixator (PC-Fix), addressed the shortcomings of the conventional plating by Dynamic Compression Plate (DCP) discovered either by careful examination of the clinical complications or by chance observation and informed inquiry in experimental animals. The focus was on avoiding iatrogenic damage to bone vascularisation caused by the implant design and mechanical function and, thus, aiding efforts of surgeons to preserve vital bone tissue needed for healing. Infections have been and will remain a great concern in all surgeries. Preservation of blood perfusion of traumatised bone is of paramount importance to reduce the risk of infection, especially in view of the emergence and the accelerated spread of bacterial resistance to antibiotics. Prof. Stephan Perren led this project in all its stages with his unique insight and wisdom. Unfortunately, due to the complex interplay of factors guiding the interests of the AO Foundation and its commercial partners, the findings of the PC-Fix project became watered down with implant systems that followed the DCP. The message of "keep the perfusion" faded into "lock the screws". The potential benefits of PC-Fix have been lost for millions of trauma patients.
\end{abstract}

Keywords: Fracture healing, fixation, fracture plating, bone healing.

*Address for correspondence: Stephen Bresina, Scyon Orthopaedics AG, Hardturmstrasse 103, CH-8005, Zurich, Switzerland.

Telephone number: +41 438330311 Email: stephen.bresina@scyon.ch

Copyright policy: This article is distributed in accordance with Creative Commons Attribution Licence (http://creativecommons.org/licenses/by-sa/4.0/).

\begin{tabular}{|c|c|c|c|}
\hline & List of Abbreviations & TCP & tri-calcium-phosphate \\
\hline & & THORP & titanium hollow screw reconstruction \\
\hline $\mathrm{AO}$ & Arbeitsgemeinschaft für & & plate \\
\hline & Osteosynthesefragen & TPLO & tibial plateau levelling osteotomy \\
\hline ADI & AO Development Institute & & \\
\hline ALPS & Advanced Locking Plate System & & \\
\hline ARI & AO Research Institute & & Introduction \\
\hline CFU & colony-forming unit & & \\
\hline c.p. & commercially pure & This con & cation in honour of the late Prof. \\
\hline DCP & Dynamic Compression Plate & Stephan & is a partial recount of the time we \\
\hline DCU & Dynamic Compression Unit & spent wit & at the ARI, Davos, and at the Maurice \\
\hline FEA & finite element analysis & Mueller & te, Bern, collaborating on what has \\
\hline IP & intellectual property & become 1 & as the PC-Fix. Only a small part of \\
\hline LCDCP & Limited Contact Dynamic & the long & mplex story of the PC-Fix project is in \\
\hline & Compression Plate & the publi & ain and these few pages will certainly \\
\hline LCP & Locking Compression Plate & not fill th & d. A long time after the books were \\
\hline LD50 & $50 \%$ incidence of infection & closed or & oroject by the $\mathrm{AO}$ Foundation and its \\
\hline PC-Fix & Point Contact Fixator & commerc & rtners, all of whom have been taken \\
\hline PCLP & Point Contact Locking Plate & over by $\mathrm{t}$ & se multinational business of Johnson \\
\hline
\end{tabular}


\& Johnson, the PC-Fix project is back on track to bring to clinical use the concepts of fracture treatment embodied in the features of the PC-Fix device. This is being realised with the ALPS-II plating system for veterinary use (Kyon AG, Zurich) and with the PCLP plating system (Scyon Orthopaedics AG, Zurich).

Fracture healing was a central interest of Prof. Perren throughout his long and productive career in basic and applied research, in trauma surgery and in medical education. Surgical professionals worldwide are indebted to him for his unparalleled leadership of the AO Foundation for almost three decades in both its research and educational arms. Our collaboration with him covers about half of that time (Slobodan Tepic's from 1983 to 1996; Steve Bresina's from 1987 to 2000). We were privileged to enjoy his encouragement and full support in many different areas of our personal, wide-ranging interests, surgical fracture treatment being but one of them.

In his major review published by the Journal of Bone and Joint Surgery in 2002, Prof. Perren provided a detailed, extensive account on the state of the art in internal fixation of bone fractures, with a comprehensive list of references covering basic biological concepts and clinical practice (Perren, 2002). Our personal views are much narrower, mostly constrained to the R\&D activities carried out at the ARI, with only echoes reaching us from the vast organisation of the $\mathrm{AO}$ and its commercial partners.

\section{Background}

Of some interest, as an anecdotal introduction to the business side of the trauma industry, was one of the ARI assignments from the AO commercial partners (at that time Mathys, Straumann and Synthes US) that S. Tepic was asked to help with during the early days of his tenure in Davos. As the patent protection of the DCP was ending, a competitor of Synthes introduced to some of its markets what seemed to be a copy of the AO DCP. Was it infringing on the still valid DCP patent? With some technical help of the Straumann Institute in Waldenburg, Slobodan's analysis showed not only that the copy plate was infringing on the patent claims but that it was an exact copy of the AO DCP. Patent attorneys of Synthes had strong enough arguments to have this copy removed from the market without going to court.

This episode carried a clear message: the DCP was crucially important to the $\mathrm{AO}$ in all its activities from its strict, structured education on fracture treatment (and the clinical practice of the tens of thousands of surgeons applying AO teachings in their daily work) to the business of its commercial partners. The DCP was by all measures the most treasured trademark of the alliance between the $\mathrm{AO}$ and the companies that have been supporting it from its inception. The reward for royalties paid to the AO by its commercial partners was their control of well over half of the world's orthopaedic trauma market. Loss of IP protection for the DCP was a serious threat and the time had arrived to come up with a replacement, hopefully, with fresh IP.

\section{Research at the ARI}

The experimental research work carried out in the late seventies and early eighties by Stephan Perren and his collaborators Berton Rahn, Jacques Cordey, Ulrich Pfister, Emanuel Gautier, Mauro Vattolo and Kaspar Jörger provided the basis and the direction for the design of a new plating system. Application of plates by screwing them onto the bone was causing bone necrosis, mostly due to the interruption of the periosteal blood flow. In turn, dead bone was removed and replaced by living bone. The timing and spatial distribution of the process were documented in detail by Emanuel Gautier by fluorescent labelling of bone formation. Observed by him and confirmed by later work on the PC-Fix, this remodelling of necrotic bone often leads to local prolonged changes in morphological and mechanical characteristics of bone tissue, with potentially detrimental consequences for the strength of the whole bone.

Continued arguments over the importance of minimising iatrogenic damage to blood perfusion of the treated bone $v s$. minimising the effects of stress shielding distract from the main rationale for the PCFix, which has always been and remains resistance to infections. In some ways, our involvement in this project received a boost from a conversation between Marc Swiontkowski and Slobodan Tepic. During their collaboration on a project at the ARI on blood perfusion of the femoral head post femoral neck fractures, Marc stated that he would never again plate a tibia fracture after having seen one that became infected. This was at the time of top excitement of using interlocked nails for all long-bone fractures. Marc's position was in a good measure the result of his training with Sigvard Hansen, one of the main proponents of interlocked nailing. Interlocked nails did turn out to greatly broaden the indications for nailing over Küntscher-type nails in long bone fractures; however, there are still hundreds of thousands of bone plates and millions of bone screws used annually in the surgical treatment of fractures.

The worldwide spread of bacterial resistance to antibiotics has created an increasing threat to medicine in general and specifically to trauma and orthopaedic surgery. The experimental work in sheep, which eventually morphed into the PC-Fix project, started with attaching to the intact tibia a device called Fixin, designed by Slobodan Tepic with the help of Franz Sutter and Oscar Tschudin (two of the most experienced and resourceful engineers at the Straumann Institute). Franz Sutter had already designed the THORP for mandibular reconstruction, which was the first locking plate system of the $\mathrm{AO}$ (Vuillemin et al., 1988). The screw heads of the THORP system were cylindrical and slotted, expanded by an 
additional small set screw with a conical head. While this was mechanically appropriate for the mandible, it was not strong nor robust enough to be used on long bones. The only other locking system that we were aware of at that time was the Zespol (Ramotowski et al., 1991), which by its basic design concept was an adaptation of an external fixator to be used internally. With its completely new surgical technique and several other practical constraints, it has found only limited clinical use.

The plate designed for the experiment in sheep was fixed to the bone using screws with conical heads that would expand a slotted ball, preinstalled in the spherical plate holes (Fig. 1). Using K-wires as temporary spacers to hold the plate off the bone during tightening of the screws resulted in a gap of 1-2 $\mathrm{mm}$ between the bone and the plate, causing minimal damage to the periosteum. Compared to all previous experiments involving sheep tibiae, histology of these tibiae stained with pre-terminally

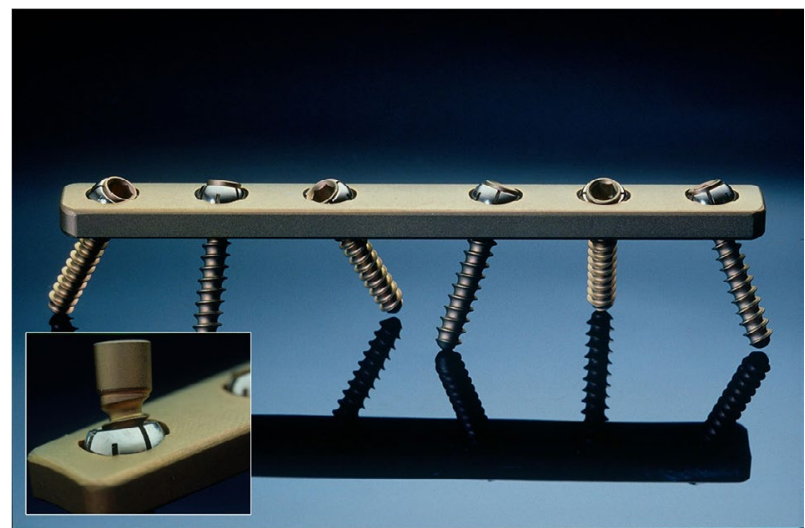

Fig. 1. Fixin - an experimental plate with locking screws (ARI).

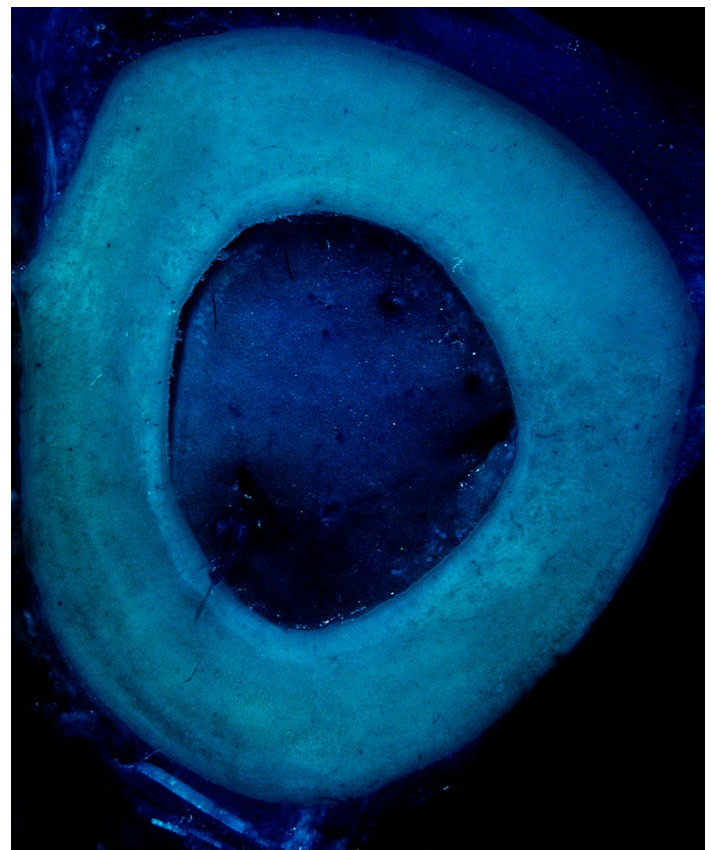

Fig. 2. A transverse section of the sheep tibia diaphysis cut between the screws of Fixin. Perfused bone is stained by pre-terminal infusion of disulphine blue 11 weeks post-operatively (ARI). infused disulphine blue, clearly demonstrated a great reduction of insult to blood perfusion by the plate (Fig. 2). However, some of the histological sections showed areas of perfusion disturbance caused by the long screws hitting larger, endosteal blood vessels (Fig. 3). Cumulative remodelling map of the bones 11 weeks postoperatively (Fig. 4) caused by perfusion disruption was produced by overlying 49 transverse sections cut between the screw holes and near the ends of the plate (7 sections of 7 tibiae) of resorption activity recorded by hand under microscopical examination. The medial aspect of the tibia where the plate was applied was on top and there was more resorption there than around the rest of the cortex but much less compared to historically observed

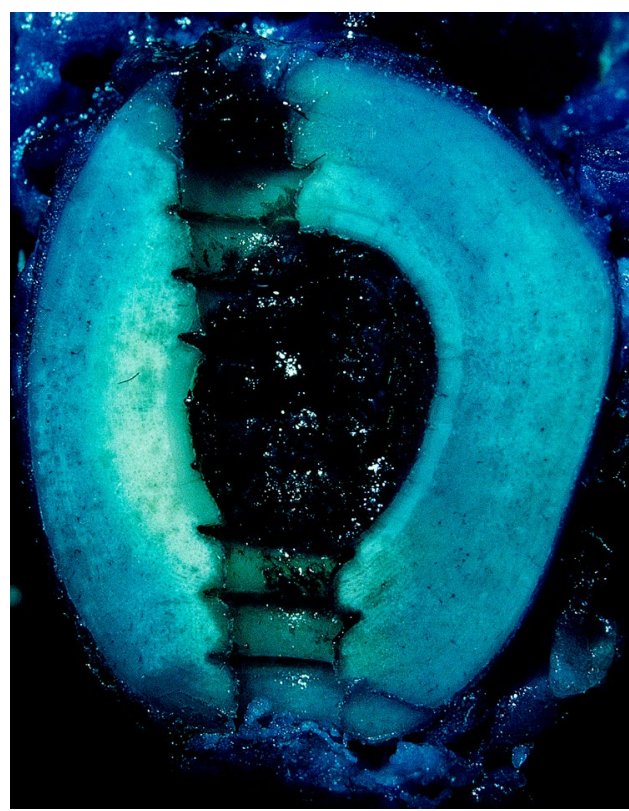

Fig. 3. A transverse section cut at the position of a screw. Lack of stain in the cortex adjacent to the screw shows disruption of the endosteal blood perfusion by the long screw (ARI).

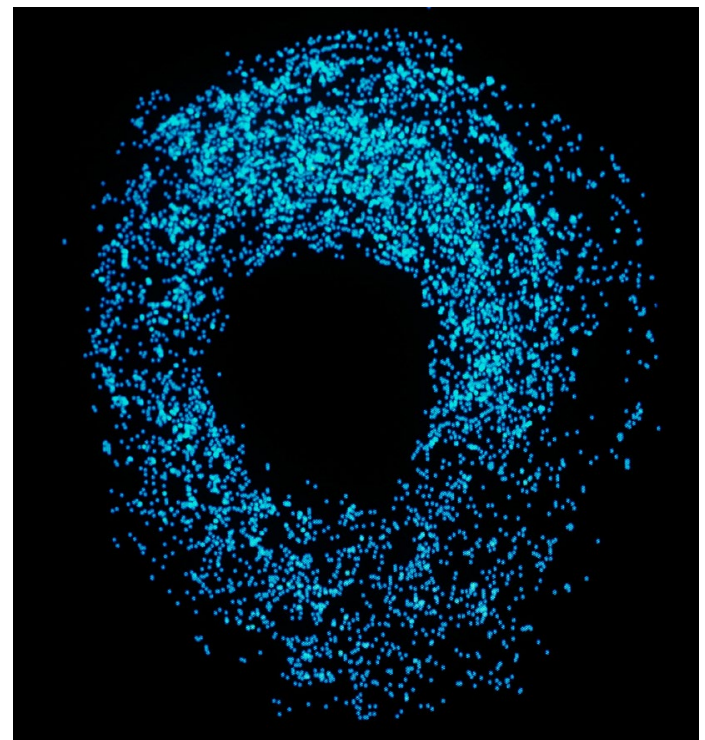

Fig. 4. A map composed from 49 overlayed transverse sections cut between the screw holes from 7 tibiae showing areas of visible bone resorption (ARI). 
massive bone loss under DCP plates. These results were not published in peer-reviewed journals. Still, the observation of bicortical screws-related damage to endosteal blood perfusion was clear enough to motivate the decision to switch to monocortical screws. This decision was a real milestone in that the mechanical features of the plate and the screws could be greatly simplified eliminating the need for angulating the screws and the locking ball of the Fixin design. The screw needs to be angulated mostly by judging the location of the exit hole in the far cortex. With the monocortical screw, this is simply not an issue - either there is good bone right under the plate in which to drill and insert the screw or there is not.

The question was if monocortical screws were sufficient for the job. From historical experience, it was clear that conventional screws fixed only in the near cortex would not suffice. However, these monocortical screws were locked in the plate and could not be "wobbled" out of the bone. The "wobbling" of the bone screws leading to bone resorption was beautifully demonstrated by Stephan Perren's earlier experiments. Mechanical experiments supporting the benefits of locked screws gave positive results (Tepic and Perren, 1995) and so in vitro tests with real and plastic bones (Miclau et al., 1995) but in vivo validation was still missing. This was the start of the PC-Fix project that ultimately - over many years resulted in experimental treatment of about 250 sheep (with an oblique or transverse tibia fracture model, with up to 2 years of follow-up) and over 400 rabbits (in the infection studies) at the ARI and at Karolinska Institute, Huddinge University Hospital, Sweden. Some of the studies were comparative, with the DCP and LCDCP used as controls, others have addressed issues of fracture model and fixation using only the PC-Fix.

The main experimental arm of the project compared the healing of oblique fractures in sheep tibiae, lagged with a $3.5 \mathrm{~mm}$ screw and neutrally plated by either a DCP or a PC-Fix on the medial side. The plates were c.p. titanium. The DCP was $12 \mathrm{~mm}$ wide and the PC-Fix was $11 \mathrm{~mm}$ wide. 7 titanium $4.5 \mathrm{~mm}$ conventional bicortical screws were used for the DCP and locked monocortical screws for the PC-Fix. The plate was fixed to the medial side of the tibia to protect the lag screw inserted in the cranial to caudal direction. The monocortical screws used for the PC-Fix were conventional $4.5 \mathrm{~mm}$ cortical screws with the heads machined to a conical shape with a standard Morse taper of $1: 20$. The fracture model was developed specifically for this study and it resulted in very consistent oblique tibia fractures in about 200 sheep at the ARI and more in other research institutions. There were 6 animals per group and the follow-up periods were 12, 24, 48 and 96 weeks. For all mechanical testing, the intact contralateral tibia was used as a control.

The results of this main study were published (Tepic et al., 1997). Briefly, in all cases, the plates were removed from the bones before mechanical testing. With bone ends potted, they were tested to failure in 4 point-bending mode, with the medial cortex with the screw holes on the tension side. This is an important detail since there were other studies performed where bones were tested with the tibia turned at $90^{\circ}$ so that the screw holes were in the neutral plane. Many of those tests showed bone strength recovery to above $100 \%$ of the intact controls. The bending strength of a no healed tibia at any time point tested immediately after the plate removal reached $100 \%$ of an intact control. The highest means reached for groups of 6 animals were $74 \%( \pm 11 \%)$ for DCP at 24 weeks and $79 \%( \pm 13 \%)$ for PC-Fix at 48 weeks. Bones tested over as close to the full length as possible failed at either the screw hole or the original fracture. Intact tibiae failed at the distal end of the diaphysis. For calibrating the test results after different periods of healing, 7 holes were drilled for screws and tapped in fresh cadavertibiae and compared to intact contralateral bones. All of those failed at the most distal hole with a mean strength of $59 \%( \pm 11 \%)$ with respect to the intact bone. No tests in any plating studies were performed with screws freshly inserted or left in the screw holes. At that time, the clinical standard of plating called for the implant removal.

At a first glance, a comparison of the strengths over healing time of close to 2 years (Tepic et al., 1997) seemed to point to no relevant difference - both groups reached comparable strength levels of about $75 \%$ with respect to intact bone between 6 months and 1 year. However, a closer look revealed major differences of potentially relevant clinical impact. At the first time point of 3 months postoperatively, all the DCP-treated bones refractured through the original fracture site at only $46 \%( \pm 4 \%)$ of the strength of intact controls. In the PC-Fix group, there was no fracture through the original fracture site - all the failures were through the most distal screw hole and the strength reached the level of $65 \%( \pm 5 \%)$ with respect to intact bone, which was slightly higher than what the tibial bones presented with freshly drilled holes at the same locations (59\%).

The strength of the tibiae in both groups continued to increase with the time of healing, but in the DCP groups failures through the original fracture site continued to occur - at 24 and 48 weeks 2 out of 6 and at 96 weeks 1 out of 6 . The only failure through the original fracture site in the PC-Fix group occurred in a single case at 96 weeks and, on a careful examination, a small bone cyst was found in the medial cortex at the fracture site. After the peak strengths of $74 \%( \pm 11 \%)$ reached at 24 weeks and $79 \%( \pm 13 \%)$ reached at 48 weeks in the DCP and PC-Fix groups, respectively, the strengths of both groups declined by 96 weeks to $57 \%( \pm 5 \%)$ for DCP and $69 \%( \pm 6 \%)$ for PC-Fix. Comparing strengths for statistical significance, PCFix was significantly stronger at 12 weeks $(p<0.001)$ and at 96 weeks $(p<0.003)$. Microradiographs (Tepic et al., 1997) of the longitudinal histological sections of cortices under the plate provided a straightforward 
explanation of the difference in strength recovery between the DCP and PC-Fix. The necrotic bone under the DCP could not even start healing across the fracture until the dead bone was resorbed and replaced by new bone. With PC-Fix, the healing of the fractured cortex started early and progressed so fast that at only 12 weeks the fracture site was stronger than the bone weakened by the screw hole in the distal diaphysis.

There was another notable difference in the failures at 96 weeks in both groups. If the failure was through a screw hole, as most of them were, it moved from the most distal to either the second or even third screw hole from the distal end. To help settle the issue of stress shielding discussions (Claes, 2020; Fouda et al., 2019; Uhthoff et al., 2006), this reduction in the strength under the plate at long follow-up was most likely due to the effects of stress protection. However, there was overwhelming evidence from histological and radiographic examinations to prove that the massive effects of early porosis under the plate must be attributed to cortical remodelling in response to necrosis caused by vascular damage. Further, if the microradiographs of the transverse sections of the bones were considered (Fig. 5), consequences of the massive early bone loss under the DCP plate were still to be observed at 96 weeks and the downward trend in strength past 24 weeks resulted in the strength at the distal segment of the tibia of only $57 \%( \pm 5 \%)$.

To address the question of potential bone response to stress protection on scales below the basic bone architecture, with remodelling effected by cellular actions of osteoclasts and osteoblast, by for example modulation of the mineral content, with Prof. Perren's enthusiastic support, a special version of an ultrasonic microscope was constructed. At that time, in the late
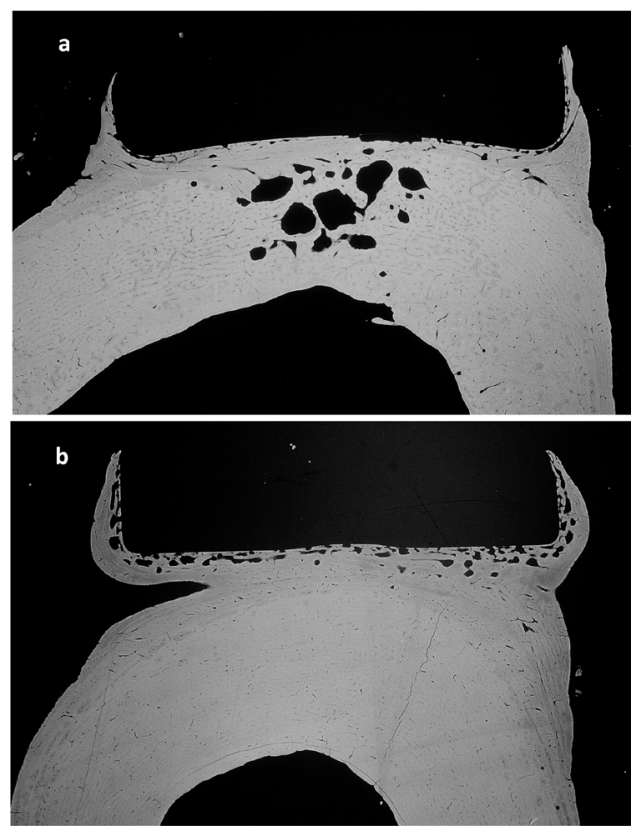

Fig. 5. Microradiographs of thin sections of cortical bone under the plates, cut between the screws of (a) DCP and (b) PC-Fix, 12 weeks post-operatively (ARI). eighties, ultrasonic microscopes were in the early stages of commercialisation, but all of them were built to examine surface and sub-surface properties only by measuring acoustic impedance, which depends on both material density and elasticity. To separate density and elasticity, our microscope had an added functionality in that it could also measure the speed of sound propagation. That was possible because the bone specimens had a known, well-defined thickness. With only a limited scope of work carried out on the bone specimens from the PC-Fix project, no clear evidence for bone "quiet" adaptation was seen on the scales examined. The acoustic impedance of a scanned cortex segment to the side of a PC-Fix plate is shown in Fig. 6. A finding of general interest to biomechanics, including reliance on radiography to judge conditions of bone tissue for clinical purposes, was that correlation of bone density and elasticity, even on a single specimen scan as shown in Fig. 6, was weak. While, in general, a higher local density correlated with a higher local elastic modulus, for a given value of density, the modulus could vary by a factor of three. We could of course clearly see the freshly deposited bone by the periosteum, which was also easily identified by microradiography or light microscopy of a labelled bone. This work on ultrasonic microscopy did not reach peer-reviewed journals.

In the original plan of the PC-Fix project, there was a separate group whereby a silicon rubber pad was to be inserted between the PC-Fix plate and the bone. Since the mechanics of plating would be very similar and made possible only with the locked screws, this would allow the effect of total disruption of periosteal blood by the silicon rubber pad (moulded to the exact shape of the underside of the PC-Fix) to be assessed. Only one sheep was treated and the cortex under the plate at 12 weeks showed massive resorption. An experiment with only one specimen of course remained unpublished.

With the DCP vs. PC-Fix study underway, a third group was added because during the project the LCDCP plate was introduced to replace the DCP. Unpublished results showed, by the outcome

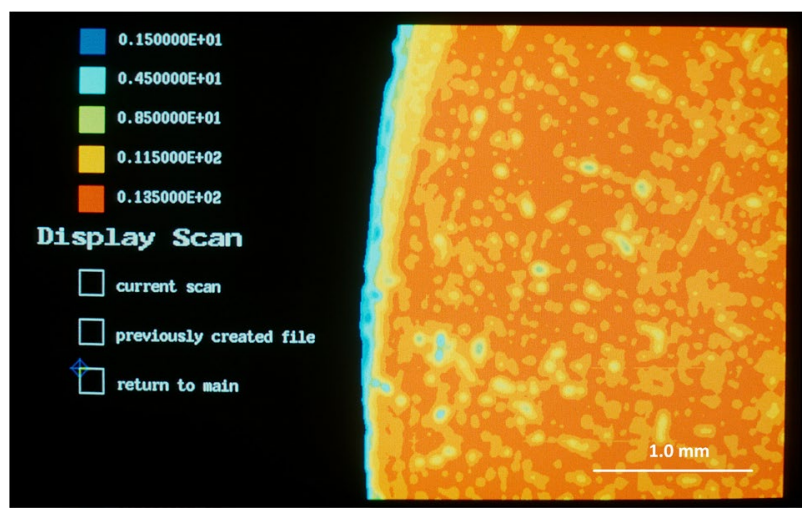

Fig. 6. An ultrasonic impedance scan of a cortex to the side of a PC-Fix plated sheep tibia, 12 weeks post-operatively (ARI). 
parameters of the study measuring the process of fracture healing, no difference between the DCP and LCDCP (the strength of the LCDCP group was lower but not significantly).

\section{Expanded animal studies}

Having observed a rapid - unexpected - strength recovery of the fractures treated using the PC-Fix by 3 months, at which point the screw holes after screw removal became the sites of critical stress concentration of approximately the same magnitude as freshly drilled screw holes, the in vivo studies were expanded using the same experimental model. At 12 weeks, the PC-Fix plates were removed and the sheep were given another 12 weeks of bone healing to estimate the strength recovery at the screw holes. The 3 study groups, each with 4 animals per group, were (i) holes left empty after plate and screw removal; (ii) holes filled with autologous cancellous bone graft; (iii) holes filled with plugs prepared from porous TCP. For the DCP and LCDCP groups, the plates were removed at 48 weeks and the bones with the screw holes (filled as for PC-Fix) given another 12 weeks of healing before testing. In the PC-Fix group, the strongest bones were those with holes left empty, $89 \%$ ( $\pm 8 \%$ ); bones with the holes filled with cancellous bone graft came second, $85 \%$ ( $\pm 14 \%$; disappointingly, TCP plugs-inhibited bone filling of the screw holes came third, $79 \%$ ( $\pm 11 \%)$; all compared to intact controls. Comparing the results by plate type, the bending strength $v s$. contralateral intact tibia in the strongest subgroups, i.e. screw holes left empty, was $89 \%$ ( $\pm 8 \%$ ) for PC-Fix, $82 \%$ ( $\pm 10 \%)$ for LCDCP and $76 \%( \pm 10 \%)$ for DCP. Given the small number of animals, there were no statistically significant differences between the groups with plates but combined with all other studies, the strength of PC-Fix-treated fractures at only 6 months being higher than that of DCP and LCDCP at 15 months had reinforced the conclusion that PC-Fix provided superior conditions for fracture healing. Unlike in the main study (Tepic et al., 1997) with testing immediately following plate removal, in this experiment, the LCDCP did show an advantage over the DCP [Foglar et al. (1994) Can filling screw holes with bioactive material decrease refracture rates? Orthop Trans J Bone Joint Surg 18: 1019, New Orleans, conference abstract].

Another similar study was performed looking at strength recovery by 12 weeks of bones weakened by screw holes but without any plate. The holes were (i) left empty; (ii) filled with bone graft; (iii) filled with TCP plugs; (iv) filled with a titanium screw. The bending strengths relative to intact contralateral controls were in decreasing sequence: cancellous graft at $75 \%( \pm 11 \%)$; empty at $72 \%( \pm 9 \%)$; TCP at $65 \%$ $( \pm 7 \%)$; titanium screw at $65 \%( \pm 5 \%)$. For calibration, freshly drilled screw holes in cadaver bones reduced the strength to $59 \%$ ( $\pm 10 \%$ ) [Frankle et al. (1993) A comparison of the effect between cancellous autologous bone graft, tricalcium phosphate, and titanium screws on empty screw holes. Orthopaedic Trauma Association, New Orleans, conference abstract].

In another major experimental animal study conducted at the ARI, Michael Schuetz compared fracture healing of sheep tibiae between the LCDCP, PC-Fix-II and an unreamed, interlocked nail up to 12 weeks (Schuetz, 2001). PC-Fix-II locking screws had a conical, threaded head, similar to the locking screws of LCP. With more parameters to monitor the healing progression, but also including mechanical testing of healed bones for strength in bending at the end of the study, the outcome, in short, was that PC-Fix-II and the unreamed nail performed equally well and were statistically superior to LCDCP.

We are unaware of any attempt at routine, very early plate removal in the clinical practice. It also seems that in most fractures currently treated, the plates are not even removed unless there are specific indications to do so. Bones were not tested with the plates and screws in situ in any of the studies but, considering all the results listed above, it is possible to assume that the bone strength even with the plate and screws in place was unlikely to attain its nominal strength. In fact, it may remain at 50 to $60 \%$ and still meet the demand of physiological function. The work of Lanyon, Rubin and collaborators in the seventies and early eighties (Rubin and Lanyon, 1982; Rubin and Lanyon, 1984) on bone surface strains measured in vivo in many animal species established the limit of 2,000 to 3,000 $\mu$-strain under peak physiological loading and 1,000 to 1,500 $\mu$-strain during average locomotion activity. Cortical bone ultimate strain in vitro is more than $6,000 \mu$-strain (Carter, 1978) so bones have at least a factor 2 safety against single cycle overload at peak activity and perhaps a factor 4 to 6 safety for an average activity such as slow locomotion.

The data collected during our studies may provide some insights that go beyond what can be learned from clinical experience alone. Ultimately, the decisions on implant removal are left to the surgeons and their patients, informed by the overall risks involved. One should not fail to consider that healing of fractures is essential to prevent implant failures - no plating hardware or procedure matches the functional requirements for high cycle fatigue strength of long bones. Unless the fracture heals, the implants will eventually fail.

\section{Development of the PC-Fix system}

As experimental evidence for PC-Fix advantages was emerging, some time was devoted to the optimisation of the plate and screw design. The geometry of the screw holes in the PC-Fix plates is much simpler and the diameter of the holes smaller than in the DCP and even more so than in the LCDCP, providing a chance to reduce the plate dimensions without compromising its strength. Initial work focused on the design of a plate equivalent to a 3.5 DCP stainlesssteel plate. The result of the optimisation using FEA 
tools was a plate of equal overall thickness but $9.5 \mathrm{~mm}$ wide instead of $10 \mathrm{~mm}$ and still about $5 \%$ stronger in bending. This was possible by making "overcuts" along the edges of the screw holes (Fig. 7), which led to a $25 \%$ reduction in the stresses relevant for fatigue endurance. This result was verified by fatigue testing. If a reduction of $0.5 \mathrm{~mm}$ in the width of the plate seemed an irrelevant detail, the increase in width from $10 \mathrm{~mm}$ to $11 \mathrm{~mm}$ and in thickness from $3 \mathrm{~mm}$ to $3.3 \mathrm{~mm}$ that the LCDCP called for should be considered. The main reason for those increases was not the strength of the plates but the reduced stiffness of now longer side walls at the plate holes. Without dimensional adjustments, material release by fretting corrosion would have doubled. Testing in patients of the early designs of the DCU, an experimental predecessor to the LCDCP, without dimensional adjustments of stainless-steel implants, confirmed the increased loss of metal and also raised the troubling prospect of tissue response to corrosion products, especially to nickel and chromium.

The design of the locking heads and screw holes of the PC-Fix was also optimised to reduce the risk of jamming, which was very low if the insertion torque was kept to a reasonable limit. The Morse cone was changed from $1: 20$ to $1: 10$, commonly used for the modular hip prosthesis. The risk of jamming without a torque-limited screwdriver is still present. However, there is a main advantage that properly locked screws offer, the elimination of fretting
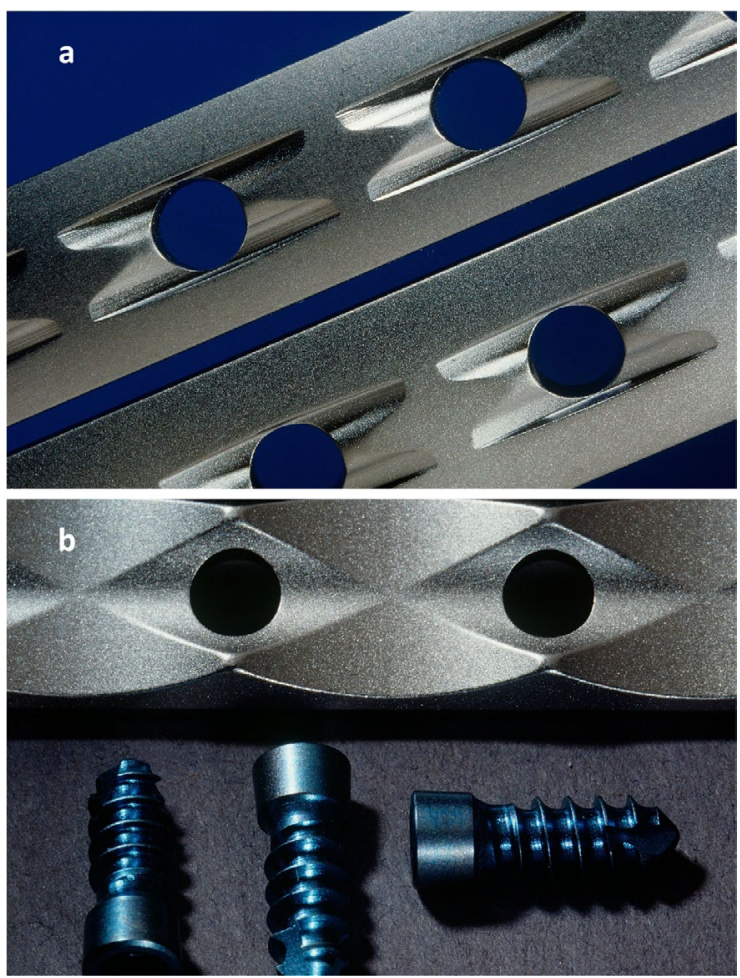

Fig. 7. PC-Fix plates. (a) Top view showing the overcuts: the upper plate is $9.5 \mathrm{~mm}$ wide, optimised with FEA and the lower plate is $11 \mathrm{~mm}$ wide. (b) Underside of the plate showing the peaks at each side of the screw hole that create the points of contact and the conical head screws (ARI). corrosion. The current instructions for use of the LCP, the state-of-the-art system from DePuy-Synthes, call for use of the conventional screws in combination with locking screws. For the initial apposition of an LCP to the bone, the use of regular cortical screws is recommended, to be followed by the insertion of locking screws.

There are two major issues with this approach. The underside of the LCP is identical to that of the LCDCP and, as already stated, there is no advantage to the LCDCP in the sheep tibia fracture healing in comparison to the DCP. The explanation is in the fact that even though the contact of the LCDCP is reduced (as implied by the name), it is still too large, especially where it really matters, i.e. over the fracture site. Therefore, the necrosis of the cortex delays the healing process. The LCP should not be expected to avoid this problem. The theoretical possibility of elevating the plate completely is annulled by practical concerns of surgical application. As shown by FEA, in the early stages of PC-Fix development, elevating the plate by only a couple of $\mathrm{mm}$ off the bone results in a very large increase in stress of both the screws and bone at the cis cortex screw hole. Secondly, fretting corrosion is only reduced but not eliminated if regular, unlocked screws are used with LCDCP or LCP. This should be of particular concern with stainless-steel implants.

\section{Bio-Logical internal fixation}

Concurrent with our technical and experimental work on plating, a growing movement in the surgical community - that came to be recognised as a BioLogical approach to internal fixation, led by Reinhold Ganz, Jeffrey Mast, Roland Jacob along with Stephan Perren - was causing internal discord within the AO. The book "Planning and reduction technique in fracture surgery" (Mast et al., 1989) was a manifesto for Bio-Logical internal fixation. Presented by some of the top experts in surgical trauma, the message of the Bio-Logical approach was considered too radical by the mainstream opinion leaders of the AO. It seemed that there was too much freedom left to the individual judgment of the surgeon that was putting at risk consistency of the outcome in pursuit of "perfect". As in most such conflicts, a compromise emerged, but unfortunately, in many cases by paying only lip service to Bio-Logical methods. Considering our already stated position of remote monitors, we still have seen some terrible examples of misunderstood Bio-Logical fixation with the plates placed on top of the muscle tissue, crushed by compression of the plate screws, killing not only the muscle but also the bone underneath. Perhaps publishing the single case of sheep tibia fracture with a silicon rubber pad under the PC-Fix would have made some sense. Most fractures, 30 years later, are still treated with little regard to keeping the periosteum alive. The missed opportunity, from our viewpoint, was the 
failure to merge those new surgical approaches with a new system of implants, exemplified by the PC-Fix. The famous words of Ilizarov "aparat-rezultat" may resonate with those who had the privilege to hear him speak.

\section{A conundrum of soft tissue separation}

In the very early stages of the PC-Fix project, the first clinical experience came from the veterinary application of those implants by Prof. Pierre Montavon at the Veterinary School of the University of Zurich. He combined the PC-Fix with the minimal fixation promulgated by the Bio-Logical approach movement in about 60 fractures of long bones in medium-sized dogs. Clinical outcomes were extremely positive, but his report was rejected for publication in veterinary peer-reviewed journals. However, in analysing his preoperative and postoperative radiographs, an interesting correlation was discovered. The amount that bone segments slipped past each other at the time of fracture closely correlated with the extent of callus after the fractures healed. Minimally displaced fractures had very short callus; those that slipped by centimeters had a callus that covered that length on both sides of the fracture.

What was behind this correlation? With an assumption that the callus needed space in which to grow, this was studied in a controlled, sheep tibia fracture model with two parameters to control: gap size at the fracture and length of soft tissue separation that would be produced surgically. With 3 values for each of the 2 parameters, the study had 9 groups, with 6 animals per group [Lederer et al. (1996) Experimentelle Frakturbehandlung mit dem Point Contact Fixator (PC-Fix) - eine in vivo Studie an der Schafstibia. Hefte zu Der Unfallchirurg Springer, Berlin 353-354]. The period for healing was limited to 12 weeks. A transverse fracture in mid-diaphysis was created using a 4 point bending jig that was fixed to the bone by pins far away from the fracture site. The fracture was fixed with a PC-Fix plate only, using $3.5 \mathrm{~mm}$ monocortical screws to fix the plate on the medial side. The three gap conditions were (i) fracture compressed; (ii) fracture reduced only; (iii) $1.5 \mathrm{~mm}$ gap controlled by a spacer inserted into the fracture gap at the time of plating. The $1.5 \mathrm{~mm}$ gap would have led to the failure of the plate. Therefore, the plate was augmented by a single $4.0 \mathrm{~mm}$ external fixator rod bridging the plated tibia on the medial side. Instead of separate bone pins, the locking screws at both ends of the plate had extensions in the form of $4.0 \mathrm{~mm}$ pins to be clamped to the rod. Soft tissue separation was obtained by the passage of a Teflon strip around the bone on each side of the fracture but above the periosteum. Separations were $0 \mathrm{~mm}$, $15 \mathrm{~mm}$ or $40 \mathrm{~mm}$ on each side. To summarise the results of this study, the highest strength in bending vs. intact contralateral tibia was $69 \%( \pm 15 \%)$ with the $1.5 \mathrm{~mm}$ gap and the $40 \mathrm{~mm}$ tissue separation; the lowest strength at $44 \%( \pm 7 \%)$ was with the $1.5 \mathrm{~mm}$ gap and no tissue separation. Tissue separation had much less effect on the compressed and reduced fractures, which were about $60 \%$ and $50 \%$ of intact, respectively.

In combination with the main study using a lagscrew fixation and a neutral plating, these experiments have provided some valuable information:

- transverse fractures can be successfully treated by plating;

- the most consistent outcomes result from compressed fractures either by lag screws or by plate compression, but only without undue damage to vascularity;

- very fast and high recovery of strength is possible even with relatively large gaps, but without tissue separation by accident or intent, such gaps present a high risk of non-union;

- the fixation hardware must be strong enoughwithout augmentation, the plates bridging large fracture gaps are at high risk of failure.

\section{Infection studies}

With Prof. Perren's foresight, the infection studies in experimental animals were started in the early stages of the PC-Fix project. Clinical studies on infections are extremely complex, slow-progressing even if multicentre, yet prone to many issues of controlling conditions between participating clinics, patients, fracture types and surgeons' preferences.

The first experimental model chosen involved a local challenge to infection in a plated rabbit tibia without a fracture by administering intraoperatively a calibrated number of CFU of Staphylococcus aureus directly to the plate site through a cannula positioned with its tip near the plate, passed through the skin incision and removed after inoculation. There were two main studies conducted at the ARI, Davos, in collaboration with the Department of Microbiology, University Hospital, Bern: (i) comparison of DCPtype plates made in medical-grade metals - 316L stainless steel or c.p. grade titanium (Arens et al., 1996); (ii) comparison of DCP to PC-Fix plates, both made in c.p. titanium (Eijer et al., 2001).

Briefly, the implants were affixed to the medial tibia cortex of rabbits with either 6 (in the first study of DCP) or 4 monocortical screws (in the second study of DCP vs. PC-Fix). After 4 weeks, the animals were euthanised and separate bacterial cultures were grown with the plates, the soft tissue and the bone beneath the plates (crushed in a bone mill). Positive cultures with bone specimens or plates were considered the most important finding for infection. The inoculum size was varied between $4 \times 10^{3}$ and $4 \times 10^{6} \mathrm{CFU}$, in a statistically guided search for the LD50. Using a grouped sequential up-and-down procedure, most of the animals were tested in the range of $4 \times 10^{4}$ to $2 \times 10^{5} \mathrm{CFU}$. The material advantage of titanium vs. stainless steel, as measured by the number of CFU to cause LD50 bone infection was a factor 10 , i.e. it took 10 times more bacteria to result 
in an LD50 incidence of bone infection with titanium than with stainless steel $\left(2 \times 10^{4}\right.$ for stainless steel $v$. $2 \times 10^{5}$ for titanium). Overall infection incidence, for all inoculum levels, for stainless steel DCP was $75 \%$, significantly higher $(p<0.05)$ than for titanium DCP (35\%). Comparing PC-Fix to DCP, both in titanium, the advantage measured by the same criterion of inoculum size for LD50 was another factor of 10 (Eijer et al., 2001). An estimate for combined effects of the plate geometry and material from the two studies

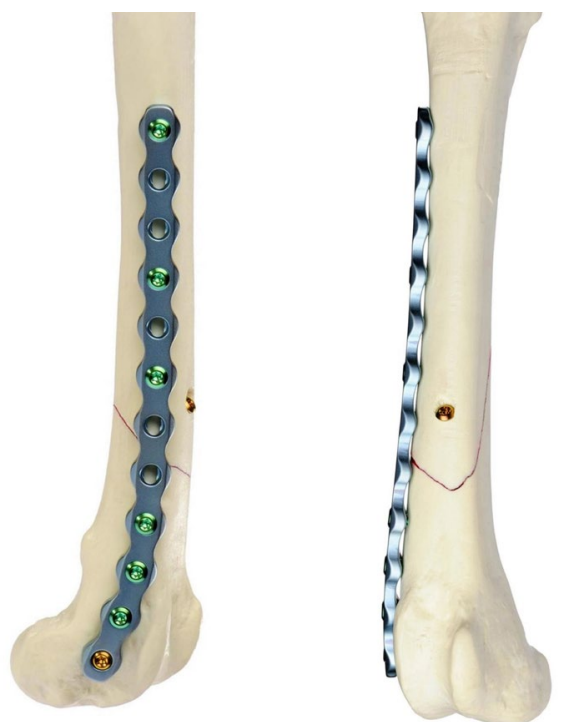

Fig. 8. Lateral and cranial views of an ALPS-10 plate on the lateral cortex of a plastic bone model of a dog femur. An oblique fracture is fixed with a 2.7 lag screw (Kyon).
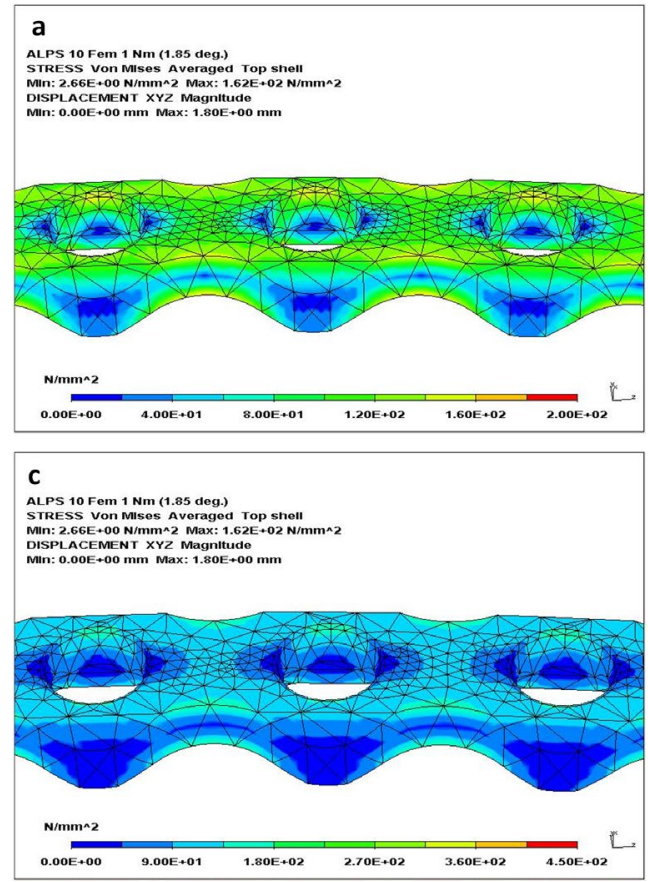

might suggest a factor of 100 higher bacterial load needed to reach LD50 infection rate in a titanium PCFix treated bone compared to a stainless steel DCP.

Is this clinically relevant? Most likely, yes. Infection rates with clinical signs are much lower than $50 \%$ but an interesting study conducted at the Veterinary School, UC at Davis (Smith et al., 1989) found positive cultures from swabs taken under stainless-steel plates at the time of plate removal in $42.5 \%$ of cases in the absence of any clinical signs. For comparison, in surgeries where no implants were used the positive tests were found in only $6.7 \%$ of cases.

Another interesting report is on bacterial DNA presence in $42 \%$ of fracture callus tissue specimens even when no isolates were found (Szczesny et al., 2008). Preventing even such stealth infections may reduce the risk of potential late outbreaks with clinical consequences. Another two infection studies were performed by Urban Lindgren and his associates at the Karolinska Institute, Huddinge University Hospital, Sweden. The first study used an experimental rabbit model with a systemic bacterial challenge (Johansson et al., 1999a), originally developed to study risks of late infections associated with hip replacement surgery. The second study utilised a local challenge by delivering bacteria close to the plates (Johansson et al., 1999b). Briefly, the first study compared infection rates of the rabbit tibia bone with plates affixed with 4 screws to the distal, mediocaudal aspect of the bone in response to systemic, intravenous challenge by Staphylococcus aureus. The inoculum was in the range from $1 \times 10^{8}$ to $2 \times 10^{9} \mathrm{CFU}$.
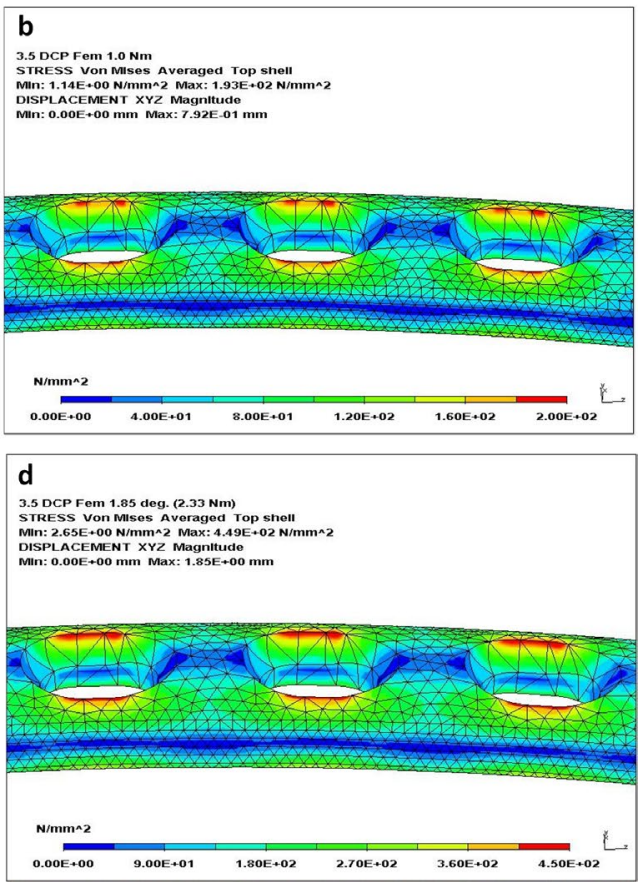

Fig. 9. Shape optimisation of ALPS by FEA. Upper row: comparison of Von Mises stresses under equal bending moments. (a) Titanium ALPS-10 plate, (b) stainless-steel DCP-3.5 plate. Maximum stress in DCP is $20 \%$ higher. Lower row: comparison of Von Mises stresses under equal displacement (angulation). (c) Titanium ALPS-10 plate, (d) stainless steel DCP-3.5 plate. DCP stress is 2.8 times higher than PC-Fix (due to lower modulus of elasticity of titanium, its deformation tolerance is higher) (Kyon). 
The plates were stainless-steel DCP, titanium DCP or titanium PC-Fix. The fourth group was with sham surgery, without implants. 111 rabbits were used in the study in these 4 groups with different numbers of animals for different inoculation levels. The largest, significant difference in infection rates was found between the PC-Fix and stainless-steel DCP with inoculates $>2.0 \times 10^{8}$ with the infection rates of $67 \%$ for DCP and $7 \%$ for PC-Fix $(p<0.01)$. In the sham surgery group, the rate was $10 \%$. Titanium DCP rate was intermediate at $33 \%$.

In the second study with the local inoculation of Staphylococcus aureus, 30 rabbits were divided into 3 groups: stainless steel DCP, titanium PC-Fix and sham surgery. The infection rates were not statistically different between the DCP and PC-Fix (60\% and $50 \%$, respectively), with the sham surgery at only $10 \%$. The inoculum was in the range from $1 \times 10^{8}$ to $1 \times 10^{9} \mathrm{CFU}$, which is higher than the highest used in the Davos studies $\left(4 \times 10^{6} \mathrm{CFU}\right)$. Even with that inoculum, some of the rabbits in the Davos study developed sepsis and were euthanised. The bacterial strains used by the two laboratories were not identical and there were some minor differences in the execution of the experiments. There is no simple explanation to reconcile the different outcomes from these two studies, which points to the problem of direct comparison without standardisation of experimental methods between different institutions.

\section{Clinical study in forearm fractures}

Motivated by positive outcomes from the preclinical testing, the ADI, Davos, initiated a large multicentre study on PC-Fix, aiming at enrolling 2,000 patients with mostly forearm fractures. With the international participation of 31 clinics, that was the largest prospective clinical trial undertaken by the $\mathrm{AO}$ Foundation. There were several reports on subcohorts of patients treated by individual surgeons or at single participating clinics (Fernandez Dell'Oca
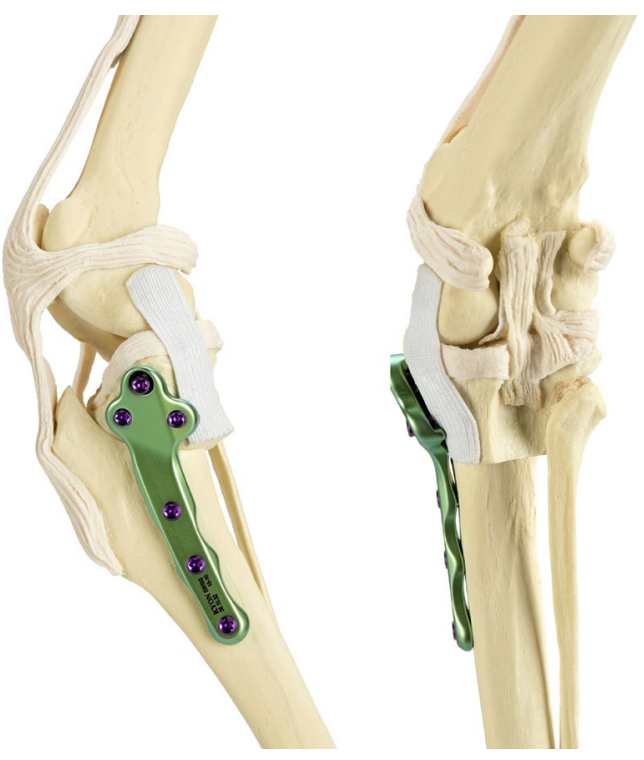

Fig. 10. ALPS ${ }^{\circledR}$ TPLO plate on a plastic model of the dog stifle (medial and caudal views) (Kyon). and Masliah Galante, 2001; Haas et al., 2001; Hertel et al., 2001). The publication referenced already in connection to an experimental animal study (Eijer et al., 2001) reported the incidence of clinical infections in 1,208 fractures treated by PC-Fix between 1993 and 1998. There were 4 infections in 256 open fractures $(1.6 \%)$ and 9 infections in 916 closed fractures $(1.0 \%)$ with 36 cases not fully documented. Thus, overall infection incidence was $1.1 \%$. There are details provided in the report on 9 infections, 4 closed and 5 open. In only one case (a closed fracture), a second surgery was performed to replace a loosened PC-Fix with an LCDCP. One additional, planned removal, was performed coincidentally with infection diagnosis. All others healed with antibiotic treatments alone. The study did accumulate close to 2,000 cases and has been used to support registration of PC-Fix in the USA (1995), Europe (1996) and Japan (1996) but the request by the $\mathrm{AO}$ Foundation for the clinical release was ignored by its commercial partners.

\section{IP and veterinary applications - ALPS $^{\circledR}$}

The main and auxiliary features of the PC-Fix system were protected by multiple patents as were those of the LCDCP (and eventually of LCP). And once again, many years after a copy of the DCP appeared shortly before the DCP patent expired, a copy of the LCDCP showed up. One of the patents for the PC-Fix was broad enough to cover some of the features of this copy of the LCDCP which its design team failed to study, having focused their attention on LCDCP patents alone. Prolonged and costly preparations for the court proceedings included deposition by S. Tepic, who meanwhile had left the ARI but was called as a witness for Synthes. Just before the court proceedings started, an agreement was reached per

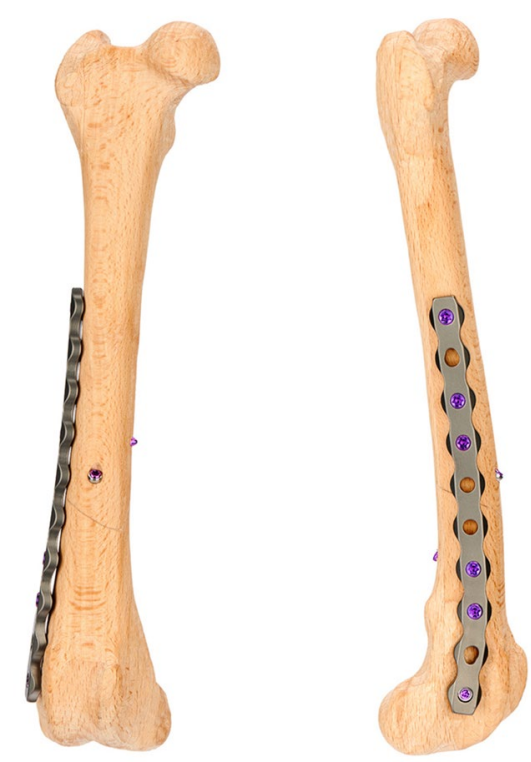

Fig. 11. ALPS-II $10 \mathrm{~mm}$ plate on the lateral side of a wooden model of the dog femur (cranial and lateral views) (Kyon). 
the request from Synthes and the copy of the LCDCP system was removed from the market. Several years later, in 2006, Synthes acquired all IP rights from the AO Foundation in preparation for one of the largest acquisitions in the industry when in 2012, Johnson \& Johnson acquired Synthes and, with that, all the IP rights generated through decades-long collaboration between the $\mathrm{AO}$ Foundation and its original commercial partners. The true irony though is that Synthes ultimately ignored the requests from the AO Foundation to commercialise the PC-Fix system and, hence, had no real use for any of the related IP other than preventing other companies from using it.

Three years after leaving the ARI in 1996, S. Tepic co-founded Kyon AG, Zurich, to design, manufacture and distribute veterinary surgical devices for orthopaedics. In 2007, the ALPS $^{\circledR}$ (Fig. 8) was introduced to the veterinary trauma market. ALPS $^{\circledR}$ emulated the main feature of PC-Fix, namely the protection of the periosteal blood perfusion, but using different screw locking mechanism and with added benefits of allowing for use in each of the holes of either a locking screw or a conventional cortical screw. The conventional screws, downsized by an increment in the diameter, could be angulated as much as in the DCP. They could also compress the fracture by slightly more than in the DCP. ALPS ${ }^{\circledR}$ plates can be contoured by bending in both planes, having the basic shape of old Sherman plates. Even with this feature, the design of the ALPS $^{\circledR}$ plates optimised by FEA, and verified by mechanical testing, resulted in about $20 \%$ higher bending strength than DCP of equal outside dimensions (Fig. 9). All implants of $\mathrm{ALPS}^{\circledR}$ are made from either c.p. titanium or titanium alloys.

With over 20,000 clinical fracture cases in dogs and cats, the basic findings from the experimental work on PC-Fix, as well as from its human trial, have been matched by the veterinary use of ALPS ${ }^{\circledR}$. There are a few publications in veterinary journals (DeTora and Boudrieau, 2016; Guerrero et al., 2014; Nojiri et al., 2015) and numerous presentations by ALPS ${ }^{\circledR}$ users at meetings of veterinary societies. Infection rates in the mix of closed and open fractures are below $0.5 \%$. In 2015 , the basic design and functional characteristics of ALPS ${ }^{\circledR}$ were applied to bone plating for uniquely veterinary treatment of the most common orthopaedic condition in dogs - cranial cruciate rupture - by TPLO (Slocum and Slocum, 1993). The AO/Synthes patents on the locking screws of PC-Fix had expired several years earlier and the screws with the conical, selflocking heads could be used in the TPLO system (Fig.
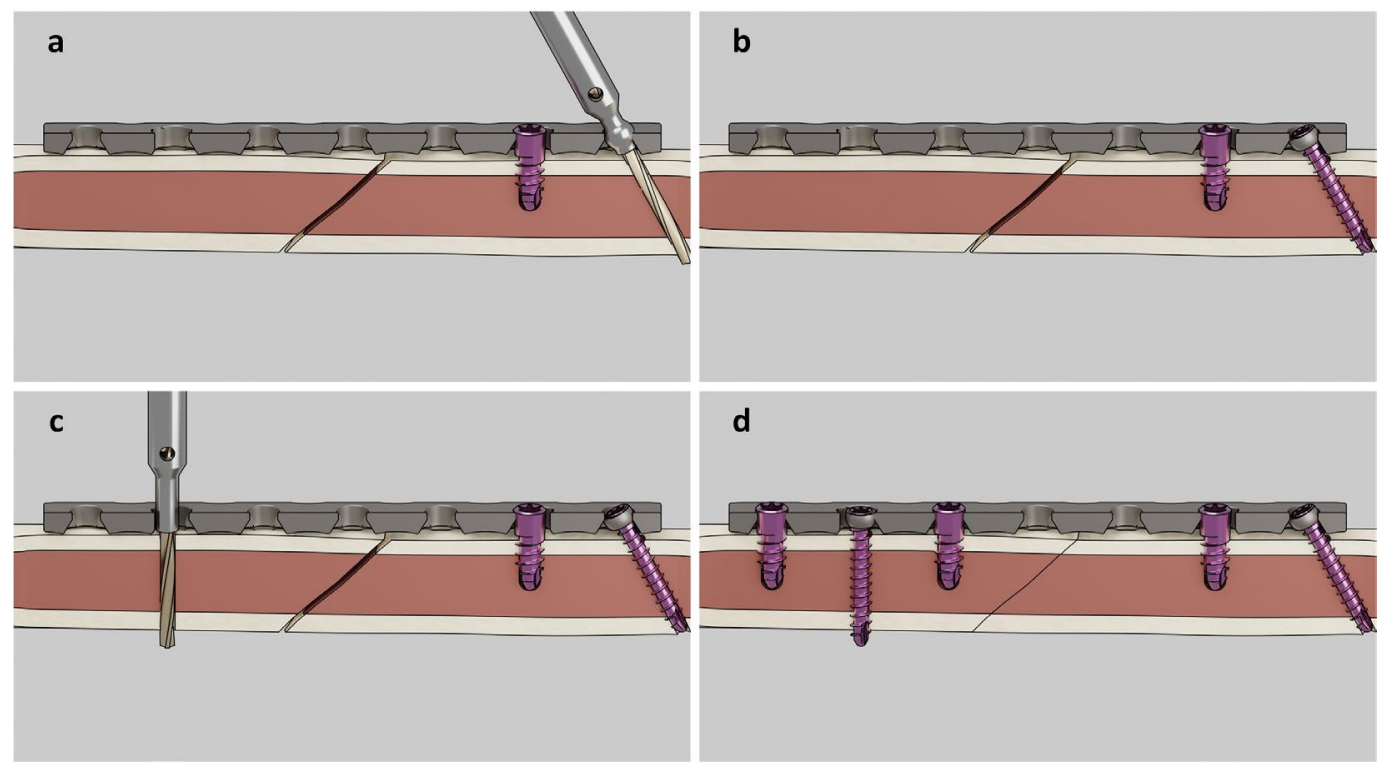

Fig. 12. Application modalities of ALPS-II. (a) Drilling for a bicortical screw into an ALPS-II plate at an oblique angle using a special drill sleeve, (b) followed by insertion of a bead and a locking screw through the bead. (c) Compression of the fracture by drilling an offset screw hole. (d) A locking screw with a bead converts the conical head into a spherical head for creating compression in the offset hole, followed by placement of monocortical locking screws (Kyon).

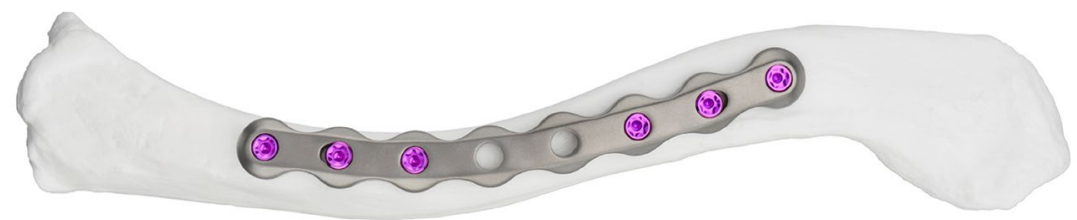

Fig. 13. PCLP plate bent in both planes affixed to a human clavicula (Scyon). 
10). In response to the fast acceptance of the TPLO, in 2019, ALPS-II was introduced with the goal of unifying the screw systems across the trauma and orthopaedic product lines of Kyon (Fig. 11). That has brought ALPS $^{\circledR}$ even closer to PC-Fix, its predecessor. But some important advantages of ALPS-II over PCFix have been maintained - namely allowing for the use of long, bicortical screws when indicated, with freedom of angulation and compression. The solution was found in using beads with downsized locking screws (Fig. 12). The beads are inserted in the screw holes after drilling of the holes (with angulation controlled by a special drill sleeve and with the range matching that of the DCP) before the locking screws are inserted and tightened, at once locking the screws in the beads and the beads in the conical holes of the plate.

\section{PCLP}

With now 14 years of ALPS $^{\circledR}$ clinical experience and with ALPS-II in its second year on the veterinary market, the PCLP system for human use, with only minor modifications to ALPS-II is being brought to the market. The system is based on $3.5 \mathrm{~mm}$ locking screws, with the plate of outside dimensions equal to the old DCP-3.5 plate intended mainly for forearm fractures. However, the option of bending in both planes may find good applications for treating fractures of bones other than the radius and/or ulna. Shown in Fig. 13 is a $10 \mathrm{~mm}$ wide PCLP affixed to a plastic clavicula.

\section{Concluding remarks}

Being always eager to move to solve the next problem, we have given insufficient attention and time to support peer-reviewed publishing of some of our work, presented here more as a narrative of events at the ARI related to this unique project than a rigorous scientific or medical discourse on fracture treatment. However, the results of the research work and development done within the unique environment created by the AO Foundation at the Davos Institutes, but also internationally, did reach a very large, worldwide audience of trauma surgeons through the AO educational programs of unparalleled scale. This summary may remind many readers familiar with the acronym PC-Fix of the years of enthusiastic efforts that brought it to their attention and, hopefully, inspire the younger generations of surgeons and researchers in related fields to tackle many unresolved problems of surgical fracture treatment.

\section{Acknowledgments}

Authors of this account of the PC-Fix project are indebted to many of their collaborators who have worked as members of different teams over that decade-long endeavour. Many have contributed to multiple aspects of the project and here are simply listed in alphabetic order (with possible omissions in view of 25 years lapsed since): Stefan Arens, Henk Eijer, Christian Foglar, Edward van Frank-Hasnoot, Christian Hauke, Markus Hehli, Mark Frankle, Robert Frigg, Maximilian Lederer, Urban Lindgren, Kurt Lippuner, Pierre Mainil-Varlet, Andreas Meiser, Ted Miclau, Gilbert Monney, Pierre Montavon, Keizo Morikawa, Miljenko Plavljanic, Marco Predieri, Andreas Remiger, Urs Schlegel, Regula Vogel and Alex Zehnder. All of us are grateful for the input of his ideas, his unconditional support and the mentoring role that Stephan Perren has granted us.

S. Tepic is a founding member of SCYON Orthopaedics and a board member of Kyon.

S. Bresina is an employee of SCYON Orthopaedics and Kyon.

\section{References}

Arens S, Schlegel U, Printzen G, Ziegler WJ, Perren SM, Hansis M (1996) Influence of materials for fixation implants on local infection. An experimental study of steel versus titanium DCP in rabbits. J Bone Joint Surg Br 78: 647-651.

Carter DR (1978) Anisotropic analysis of strain rosette information from cortical bone. J Biomech 11: 199-202.

Claes L (2020) Improvement of clinical fracture healing - What can be learned from mechanobiological research? J Biomech 115: 110148. DOI: 10.1016/j.jbiomech.2020.110148.

DeTora MD, Boudrieau RJ (2016) Complex angular and torsional deformities (distal femoral malunions). Preoperative planning using stereolithography and surgical correction with locking plate fixation in four dogs. Vet Comp Orthop Traumatol 29: 416-425.

Eijer H, Hauke C, Arens S, Printzen G, Schlegel U, Perren SM (2001) PC-Fix and local infection resistance - influence of implant design on postoperative infection development, clinical and experimental results. Injury 32 Suppl 2: B38-B43.

Fernandez Dell' Oca AA, Masliah Galante R (2001) Osteosynthesis of diaphyseal fractures of the radius and ulna using an internal fixator (PC-Fix). A prospective study. Injury 32 Suppl 2: B44-B50.

Fouda N, Mostafa R., Saker A (2019) Numerical study of stress shielding reduction at fractured bone using metallic and composite bone-plate models. Ain Shams Eng J 10: 481-488.

Guerrero TG, Kalchofner K, Scherrer N, Kircher P (2014) The Advanced Locking Plate System (ALPS): a retrospective evaluation in 71 small animal patients. Vet Surg 43: 127-35.

Haas N, Hauke C, Schütz M, Kääb M, Perren SM (2001) Treatment of diaphyseal fractures of the forearm using the Point Contact Fixator (PC-Fix): results of 387 fractures of a prospective multicentric study (PC-Fix II). Injury 32 Suppl 2: B51-B62. 
Hertel R, Eijer H, Meisser A, Hauke C, Perren SM (2001) Biomechanical and biological considerations relating to the clinical use of the Point ContactFixator - evaluation of the device handling test in the treatment of diaphyseal fractures of the radius and/ or ulna. Injury 32 Suppl 2: B10-B14.

Johansson A, Lindgren JU, Nord CE, Svensson O (1999a) Material and design in haematogenous implant-associated infections in a rabbit model. Injury 30: 651-657.

Johansson A, Lindgren JU, Nord CE, Svensson O (1999b) Local plate infections in a rabbit model. Injury 30: 587-590.

Mast J, Jacob R, Ganz R (1989) Planning and reduction technique in fracture surgery. SpringerVerlag, Berlin.

Miclau T, Remiger A, Tepic S, Lindsey R, McIff T (1995) A mechanical comparison of the dynamic compression plate, limited contact-dynamic compression plate, and point contact fixator. J Orthop Trauma 9: 17-22.

Nojiri A, Nishido T, Horinaka O, Akiyoshi H, Ohashi F, Yamaguchi T (2015) Initial clinical application and results of the Advanced Locking Plate System (ALPS) in small animal orthopedics: two hundred eighty two procedures. Int J Appl Res Vet M 13: 64-79.

Perren SM (2002) Evolution of the internal fixation of long bone fractures. The scientific basis of biological internal fixation: choosing a new balance between stability and biology. J Bone Joint Surg Br 84: 1093-1110.

Ramotowski W, Granowski R. (1991) An original method of stable osteosynthesis. Clin Orthop Relat Res 272: 67-75.

Rubin CT, Lanyon LE (1982) Limb mechanics as a function of speed and gait: a study of functional strains in the radius and tibia of horse and dog. J Exp Biol 101: 187-211.

Rubin CT, Lanyon LE (1984) Dynamic strain similarity in vertebrates; an alternative to allometric limb bone scaling. J Theor Biol 107: 321-327.

Schuetz MA (2001) Neue Stabilisierungskonzepte mit Fixateur intern Systemen bei der Versorgung von Frakturen langer Röhrenknochen - Experimentelle und klinische Grundlagen. Habilitationsschrift. Medizinischen Fakultät Charité der HumboldtUniversität, Berlin.

Slocum B, Slocum TD (1993) Tibial plateau leveling osteotomy for repair of cranial cruciate ligament rupture in the canine. Vet Clin North Am Small Anim Pract 23: 777-795.

Smith MM, Vasseur PB, Saunders HM (1989) Bacterial growth associated with metallic implants in dogs. J Am Vet Med Assoc 195: 765-767.

Szczêsny G, Interewicz B, Swoboda-Kopeć E, Olszewski WL, Górecki A, Wasilewski P (2008) Bacteriology of callus of closed fractures of tibia and femur. J Trauma 65: 837-842.

Tepic S, Perren SM (1995) The biomechanics of the PC-Fix internal fixator. Injury 26 Suppl 2: B5-B10.
Tepic S, Remiger AR, Morikawa K, Predieri M, Perren SM (1997) Strength recovery in fractured sheep tibia treated with a plate or an internal fixator: an experimental study with a two-year follow-up. J Orthop Trauma 11: 14-23.

Uhthoff HK, Poitras P, Backman DS (2006) Internal plate fixation of fractures: short history and recent developments. J Orthop Sci 11: 118-126.

Vuillemin T, Raveh J, Sutter F (1988) Mandibular reconstruction with the titanium hollow screw reconstruction plate (THORP) system: evaluation of 62 cases. Plast Reconstr Surg 82: 804-814.

\section{Discussion with Reviewers}

Nicholas Bishop: To what extent will it be practically possible to clinically demonstrate the advantages of PC-Fix clinically, compared to conventional systems? Authors: It is highly unlikely that a proper comparative study will ever be conducted. The study supported by the AO Foundation/Synthes 30 years ago was not designed towards that end. Since the main advantage of the PC-Fix is expected to be in reducing the infection risk, looking at fractures in lower extremities would likely call for a smaller study than with forearm fractures. However, the use of interlocked nails has gained an advantage in those indications, making such a study more difficult to organise. Our hope is that observations by either single surgeons or within single institutions that may decide to try the PCLP will be able to connect what they can directly experience in surgery with what the experimental work and the clinical use have demonstrated so far. This has been the case with the veterinary surgeons using the ALPS. We like to mention one detail that everyone should be able to observe: in drilling bones for conventional, bi-cortical screws, by looking at the hole for just a very brief time, the surgeon will almost always see a lot of blood streaming out of the hole before the screw is inserted. When drilling for mono-cortical screws with a drill stop, flushing the hole with saline and waiting for any blood to come out will need much, much longer. No histology should be more convincing than this observation in demonstrating the damage done to endosteal bone perfusion - bleeding is a sure sign that some bone will end up dying increasing the risk of bacteria establishing their foothold.

Nicholas Bishop: Do the authors consider some interfragmentary motion to be advantageous to stimulate secondary healing or can osteonal healing be accommodated to some extent by PC-Fix?

Authors: The extensive experimental studies in animals as well as clinical veterinary use of the ALPS ${ }^{\circledR}$ have dispelled the initial concerns that reduced stiffness of titanium PC-Fix as compared to stainless steel DCP would result in reduced stability and excessive callus formation. In fact, under carefully 
controlled conditions of fracture fixation in the main experimental study comparing PC-Fix to DCP and LCDCP, the fracture callus was significantly smaller in the PC-Fix group. Those oblique fractures were fixed by a lag screw and both plate types provided sufficient protection of the lag screw fixation. The increased callus size turned out to be due to bi-cortical screws (and the drill bit before the screws) exiting the bone on the far side and creating a separation of the soft tissues. For the surgeon dealing with real-life complications of internal fixation, controlling the interfragmentary conditions is probably not possible, especially because in all cases, this motion is primarily caused by functional demands that even the patient has limited means to control. In the sheep model that we have used, there was definite histological proof that direct osteonal healing was possible with PC-Fix even though the sheep were allowed full use of their limbs within their stall boxes after only a short period of post-operative support by a sling.

Nicholas Bishop: Could the authors comment on the relative ease of surgery with a point-contact type system?

Authors: As with any fracture fixation, the main difficulty is in getting a proper reduction. Once that is accomplished by direct or indirect techniques, PCFix fixation is easier and quicker, with perhaps less freedom but also lower risk of mistakes.

Nicholas Bishop: It must be important to maintain the circularity of a locking screw hole when contouring a plate. How is this achieved?

Authors: The plates are designed with the sections between the screw holes weaker than the sections around the screw holes. This helps to maintain the shape of the holes even if the plate is being contoured over sections that include screw holes. The bending instruments (bending irons) for out-of-plane bending cover the holes and confine the highest stresses to the section between the holes (in a 4 point-bending mode). For large bends ( $>45^{\circ}$ at a single span), holeto-hole bending is recommended; for gentle bends, it is acceptable to bend the plate over several holes. The same is true for twisting the plates. Regarding the ALPS $^{\circledR}$ plates for veterinary use and the PCLP further development of ALPS $^{\circledR}$ for human use, for in-plane bending there is a special instrument that places the highest stresses between the adjacent holes - the only way possible to use this instrument is hole-tohole. Even with these precautions, the holes will not remain perfectly round if the plates are bent to the maximum allowed by the instruments. However, the screws will still lock safely and not jam unless excessive torque is used.

Reviewer 1: In addition to the PC-Fix research conducted at the ARI during your time there, what would you say have been the other major research accomplishments emanating from the ARI?
Authors: The ARI was divided into several research units and we will respond to this question by providing a list of only the projects that we were directly involved with in the area of what might be considered as biomechanics and related technologies. The names of researchers who have worked with us are given in alphabetic order - many of them have contributed their time and skillsets to more than one of these endeavours. As already stated in our report, all of these activities were approved and fully and actively supported by Prof. Perren.

1. Stereolithography machine (in its early, precommercial-phase; today this is one of the technologies known as 3D additive manufacturing) with shape optimisation to match the geometry and mechanical properties of bones (polymerisation by a UV laser).

2. 3D gel structures with post-processing to make scaffolds for tissue engineering (a unique process of selective gel melting by an infrared laser).

3. Ultrasonic microscopy with expanded functionality.

4. Computer simulations of fibre movement in a gel-like medium applied to potential orientation mechanisms of collagen networks, including in vitro studies by transmission electron microscopy of vitrified cartilage specimens to support the concept and establish key simulation parameters; demonstrations by bench-top models.

5. Spine disc mechanics based on fluid flow rectification at endplates (non-linearities of constriction flow out of the disc vs. imbibition); computer simulations verified by bench-top models and by in vitro measurements on sheep spine specimens supported by electron microscopy studies of human specimens.

6. Design and construction of a $6^{\circ}$ freedom testing machine for spine biomechanics.

7. Optimisation of hip prosthesis implants based on improved muscle force simulations.

8. Response of cancellous bone to loading - in vivo.

9. Rectification of fluid percolation in bone tissue - computer modelling and experimental verification.

10. Gel-spinning of poly(lactic acid) fibres avoiding molecular fibre skin orientation.

11. Cell-cycle modelling with applications to cancer; design and integration of an extracorporeal blood treatment machine for selective amino acid removal.

12. Improving bone cement properties, including preheating of prosthetic stems; computer simulations of heat transfer with in vitro verification studies.

13. Numerical optimisations of bone screws with mechanical testing.

14. Optimisation of bone pins for external fixation; parametric design studies; in vivo and in vitro testing focused on the pin-bone interface.

15. Fracture gap strain experimental in vivo studies in sheep, supplemented by computer simulations 
of strain distribution in the tissues surrounding the gap; bench-top model studies of fluid flow in the non-linear poroelastic gap tissue.

16. Motorised, remotely controlled, bone lengthening intramedullary nail; design, production and experimental in vivo testing.

17. External fixator for distal radius fractures compatible with wrist-kinematics; cadaver studies; clinical handling testing.

18. Biomechanics of the hip labrum - elucidation of its role in the overall function of the hip joint.

19. Computer modelling of non-linear dynamics of HIV infection with major implications for pathophysiology and potential treatments of AIDS.

20. "Compass" for osteoclasts in bone remodelling based on strain-induced fluid flow into and out of the cutting cones; computer simulations.

21. Continuously driven, motorised external fixators for bone lengthening; experimental animal testing comparing it to the conventional, manual stepwise procedure; including a successful pilot use on a human patient.

Most of these accomplishments were documented in the graduate theses of our collaborators listed below, very few in peer-reviewed journals. About half of these people have chosen academic careers and by now have become professors at engineering and medical university departments internationally.

Dana Ayotte, Tim Biliuris, Nicholas Bishop, Eduardo Crespo, Steve Ferguson, Bernd Füchtmeier, Sylwester Gogolewski, Karel Goslings, Ed von Frank Haasnoot, Reiner Hente, Klaus Hyldahl, Keita Ito, Kurt Lippuner, Daniel Martin, Terence McIff, Seija Pearson, Pawel Pyk, Geoff Richards, Patricia Seemann, Roland Steck and Melissa Tate.

Editor's note: The Scientific Editor responsible for this paper was Chris Evans. 